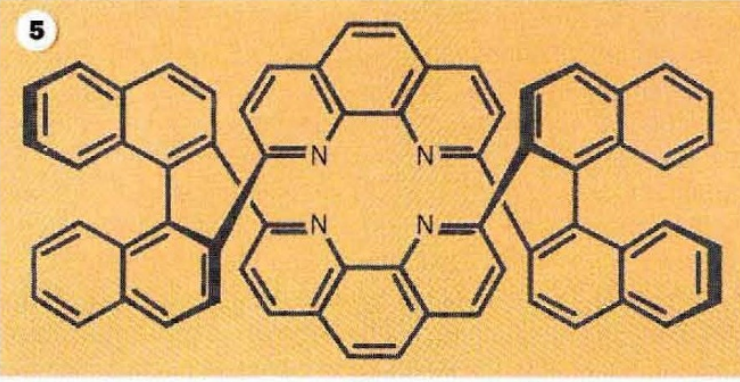

6
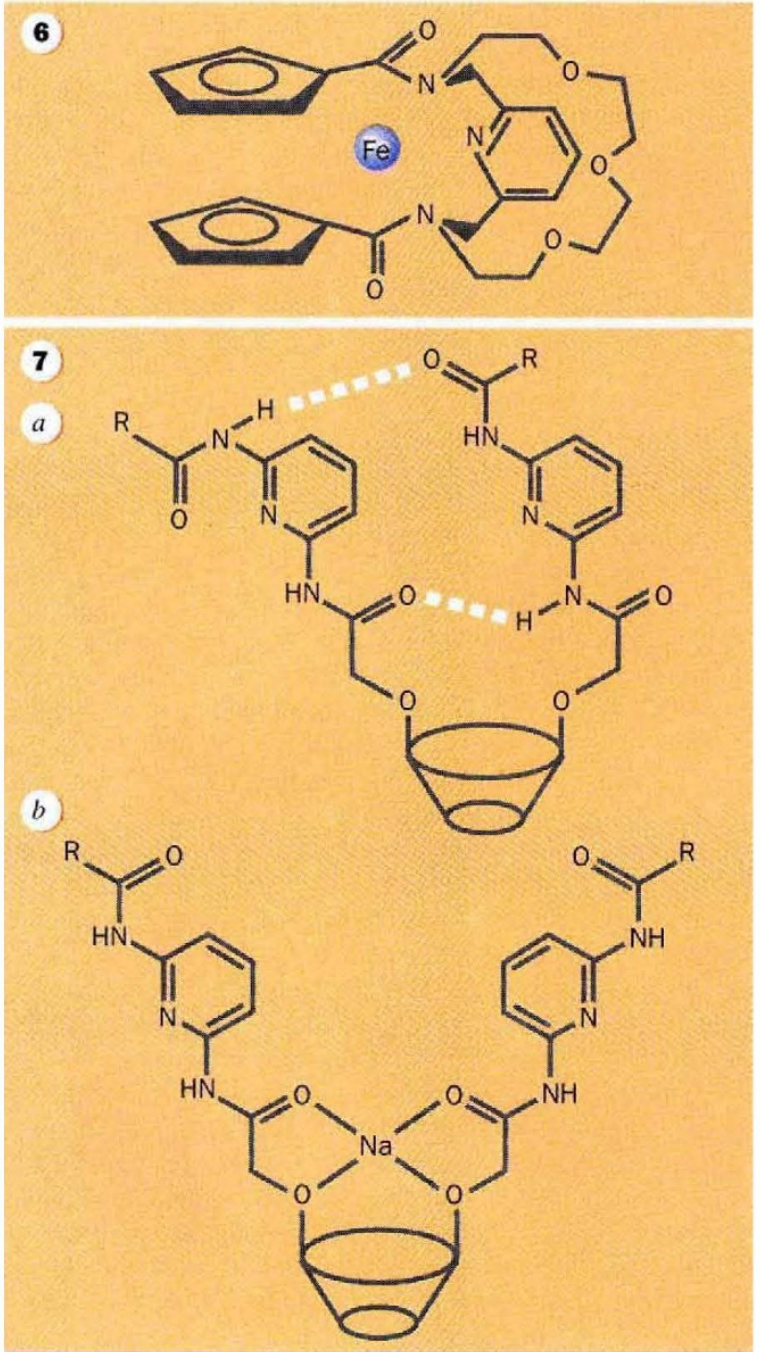

In another interesting development, Hall et al. ${ }^{10}$ have designed a molecular receptor containing a redox-active ferrocene group and a macrocycle with both pyridine nitrogen and polyether oxygen donor atoms (6). The ferrocene group acts as an electrochemical probe, and preorganizes the macrocycle for the binding of metal ions. When 6 interacts with calcium salts, the result is the assembly of a sandwiched 2:1 complex, in which the ferrocenyl groups are brought close together by the coordination of the macrocycle to the metal ion.

Finally, we may consider a functionalized calixarene reported by Murukami and Shinkai ${ }^{11}$. Calixarenes are cyclic, bucket-shaped molecules which have been widely used as host molecules in supramolecular chemistry. In this case, Murukami and Shinkai are using the calixarene as a spacer for two pyridinediamide groups, which are attached to the 'rim' of the bucket. These two pendant groups are strongly hydrogen-bonded to each other to give the 'closed' ligand $7 \mathbf{a}$. Interaction of $7 \mathbf{a}$ with sodium salts results in a conformational change to generate a binding site for the metal ion. The coordination of the sodium to two oxygen donors of each pendant chain results in a complex of the 'open' form of the ligand, $\mathbf{7 b}$. In this form, the three nitrogen atoms of each side chain are

incorporated into the components of supramolecular systems. A particularly exciting application is seen in the interactions of polypeptides modified by terminal 15-crown-5 groups with group I metal ions. Sodium ions fit snugly into the cavity of 15 -crown-5 to give $1: 1$ complexes, whereas the larger potassium cation forms 2:1 complexes in which the metal ion is sandwiched between two crown ether ligands. In the case of the modified peptide, interaction with potassium salts gives a bundle in which two $\alpha$-helical rods are linked head-to-head. Molecules of this type will allow metaldirected bundle assembly processes to be probed, and have an obvious relevance to the changes in helix-helix association state that occur in biological ion channels.
DAEDALUS

\section{Warning twinges}

LAST week Daedalus suggested that the brain is sensitive to radiation; that radioactive decay within it releases small doses of neurotransmitter at random. Oddly enough, some of the brain's neurotransmitters, such as serotonin and bradykinin, act in the body as pain transmitters. They are released from inflamed or damaged tissue, and trigger the nerves of pain.

Clearly, says Daedalus, creative evolution is working on a new pain sense to warn us against dangerous levels of radiation. To help it along, he is devising a radiation-sensitizing drug. Many enzymes are reversibly activated by changes of temperature or $\mathrm{pH}$;

DREADCO's biochemists are devising one that is activated by radiation. They are modifying the enzyme which controls the synthesis of bradykinin so that it is normally inactive, but can be flipped into its active form by the ionic track of a high-energy particle. It may then generate thousands of molecules of bradykinin, enough to launch a pulse into a pain nerve, before it flops back into its inactive form to await the next particle. (Rhodopsin in the eye is briefly flipped by visible photons in much the same way.) As one particle may create thousands of ion pairs, each of which may induce the enzymatic formation of thousands of bradykinin molecules, even a single particle might cause a brief but noticeable twinge of pain. When the new enzyme has been perfected, DREADCO will market it as 'Radiosense', the pill that lets you feel radiation.

Radiosense will usher in a new era of radiation safety. Its dosage will be chosen so that harmless or background radiation has no effect, minor warning levels are uncomfortable, while serious intensities become intolerable. It will be a wonderful liberator for physicists, X-ray technicians and nuclear-power workers. In effect, they will become their own Geiger counters: they will automatically sense every nuance of their radiation environment. Instead of obeying cumbersome and often misdirected safety rules, they will feel any problem that arises, and will be able to track it down immediately. The pain of a serious leak will force them to escape or don protective clothing, whose effectiveness or otherwise will be obvious at once. Safety will enforce itself: only a determined masochist could suffer radiation injury. Radiosense should also sell widely to ordinary citizens who are not sure how frightened they should be of $X$-rays, radon, reactors or even computer screens. The product will either reassure them, or give them solid grounds for complaint.

David Jones
Edwin C. Constable is in the Institut fur 4056 Basel, Switzerland. 